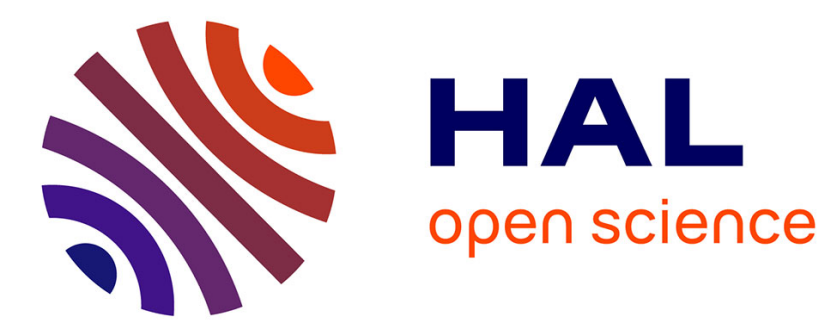

\title{
Finite-Time and Fixed-Time Observers Design via Implicit Lyapunov Function
}

\author{
Francisco Lopez-Ramirez, Andrey Polyakov, Denis Efimov, Wilfrid
}

Perruquetti

\section{- To cite this version:}

Francisco Lopez-Ramirez, Andrey Polyakov, Denis Efimov, Wilfrid Perruquetti. Finite-Time and Fixed-Time Observers Design via Implicit Lyapunov Function. European Control Conference 2016, Jun 2016, Aalborg, Denmark. hal-01298162

\section{HAL Id: hal-01298162 \\ https://inria.hal.science/hal-01298162}

Submitted on 5 Apr 2016

HAL is a multi-disciplinary open access archive for the deposit and dissemination of scientific research documents, whether they are published or not. The documents may come from teaching and research institutions in France or abroad, or from public or private research centers.
L'archive ouverte pluridisciplinaire HAL, est destinée au dépôt et à la diffusion de documents scientifiques de niveau recherche, publiés ou non, émanant des établissements d'enseignement et de recherche français ou étrangers, des laboratoires publics ou privés. 


\title{
Finite-Time and Fixed-Time Observers Design via Implicit Lyapunov Function
}

\author{
Francisco Lopez-Ramirez, Andrey Polyakov, Denis Efimov and Wilfrid Perruquetti
}

\begin{abstract}
This paper deals with the problem of finite-time and fixed-time observation of linear multiple input multiple output (MIMO) control systems. The nonlinear dynamic observers, which guarantee convergence of the observer states to the original system state in a finite and a fixed (defined $a$ priori) time, are studied. Algorithms for the observers parameters tuning are also developed. The theoretical results are illustrated by numerical examples.
\end{abstract}

\section{INTRODUCTION AND RELATED WORKS}

State estimation (observation) is one of the classical control problems [1], [2], which still forms a very active research domain [3], [4], [5]. Observer design algorithms give background for the development of fault detection [6] and data assimilation [7] systems.

Observation with time constraints (non-asymptotic observation) is important and an interesting problem for both control theory [8], [9] and practice [10]. On the one hand, finite-time observation is a simple way to realize the separation principle (the control law can be designed and analyzed independently of the observer). On the other hand, the transition processes of many control systems are strongly restricted in time. For example, the state estimate of a walking robot must be provided before each impact with the ground, i.e. calculated within the length of each step [11].

One of the most popular approaches to system state estimation is the so-called dynamic observer design, which uses a copy of the mathematical model of system with an additional output injection term (see, for example, [2], [12], [13] etc). In this approach, the observer is designed firstly by studying the stability of the differential equation that describes the observation error, then, a nonlinear output injection term can be applied in order to guarantee the finitetime or fixed-time (prescribed in advance) convergence of the observation error to zero. To attain this type of convergence, high order sliding mode [14], [15], [16] and homogeneous observers [10], [9], [3] can be used and they admit a rather simple and constructive representation. However, the

F. Lopez-Ramirez, A. Polyakov, D. Efimov and W. Perruquetti are with Non-A INRIA - LNE, Parc Scientifique de la Haute Borne 40, avenue Halley Bat.A, Park Plaza 59650 Villeneuve d'Ascq (e-mail: francisco.lopezramirez@inria.fr, andrey.polyakov@inria.fr, denis.efimov@inria.fr, wilfrid.perruquetti@inria.fr).

D. Efimov and A. Polyakov are also with the Department of Control Systems and Informatics, University ITMO, 49 av. Kronverkskiy, 197101 Saint Petersburg, Russia.

W. Perruquetti is also with CRIStAL UMR CNRS, Ecole Centrale de Lille, BP 48, Cité Scientifique, 59651 Villeneuve-d'Ascq, France.

This work was partially supported by ANR 15 CE23 0007 (Project Finite4SoS), the Government of Russian Federation (Grant 074-U01) and the Ministry of Education and Science of Russian Federation (Project 14.Z50.31.0031). practical implementation is often complicated since, to the best of our knowledge, the existence of appropriate observer parameter tuning hasn't been provided for high order systems.

The present paper develops effective computational algorithms for tuning the parameters of a finite-time observer and a novel fixed-time one, both of whom are based on homogeneity properties and the constructive procedures to adjust the observers parameters rely in Linear Matrix Inequalities (LMI) [17]. The ideas used here are a natural analog of the ones presented in [18] applied in the observation context, moreover, in the observers to be presented there is no need of online calculation of the Implicit Lyapunov Function (ILF), reducing considerably the computational requirements of its implementation.

This paper is organized as follows: The next section presents the problem statement and basic assumptions. Next the preliminary results related to finite-time and fixed-time stability and the ILF method are given. Sections IV, V and VI deal with the main results, numerical simulations and concluding remarks, respectively.

Notation: let $\lambda_{\min }(P)$ and $\lambda_{\max }(P)$ be the minimum and maximum eigenvalue of the positive definite symmetric matrix $P=P^{T} \in \mathbb{R}^{n \times n}$, respectively; $I_{n} \in \mathbb{R}^{n \times n}$ denotes the identity matrix; $\left(z_{1}, z_{2}, \ldots, z_{m}\right)$ denotes the diagonal matrix with diagonal elements $z_{i}, i=1,2, \ldots, m ; \operatorname{rown}(W)$ and $\operatorname{coln}(W)$ are the number of rows and the number of columns of the matrix $W$, respectively (i.e. if $W \in \mathbb{R}^{p \times q}$ then $\operatorname{rown}(W)=p$ and $\operatorname{coln}(W)=q) ; \operatorname{ker}(W)$ and range $(W)$ are the null space and the column space of the matrix $W$, respectively; $\operatorname{null}(W)$ is the matrix with columns defining the orthonormal basis of the subspace $\operatorname{ker}(W)$.

\section{PRoblem STATEMENT}

Let us consider the following linear control system:

$$
\left\{\begin{array}{l}
\dot{x}(t)=A x(t)+B u(t), \\
y(t)=C x(t),
\end{array}\right.
$$

where $x \in \mathbb{R}^{n}$ is the state variable, $y \in \mathbb{R}^{k}$ is the measured output, $u: \mathbb{R} \rightarrow \mathbb{R}^{s}$ is a control input, $A \in \mathbb{R}^{n \times n}$ is the system matrix, $B \in \mathbb{R}^{n \times s}$ is the matrix of input gains and the matrix $C \in \mathbb{R}^{n \times k}$ is the output matrix that links the measured outputs with the state variables. The pair $\{A, C\}$ is assumed to be observable and $\operatorname{rank}(C)=k$.

The goal is to design a dynamic observer, which estimates the state of the system (1) in a finite or a fixed (defined a priori) time under the assumption that the domain of initial conditions of the system (1) is unknown. 


\section{PRELIMINARIES}

\section{A. Finite-time and fixed-time stability}

Let us consider the system of the form

$$
\dot{x}(t)=f(t, x(t)), \quad t>t_{0}, \quad x\left(t_{0}\right)=x_{0},
$$

where $x \in \mathbb{R}^{n}$ is the state vector, $f: \mathbb{R}_{+} \times \mathbb{R}^{n} \rightarrow \mathbb{R}^{n}$ is a nonlinear continuous vector field. Let us assume that the origin is an equilibrium point of (2).

Definition 1 ([19], [20], [21]). The origin of system (2) is said to be globally uniformly finite-time stable if it is uniformly Lyapunov stable and finite-time attractive, i.e. there exists a locally bounded function $T: \mathbb{R}^{n} \rightarrow \mathbb{R}_{+} \cup\{0\}$ such that $x\left(t, t_{0}, x_{0}\right)=0$ for all $t \geq t_{0}+T\left(x_{0}\right)$, where $x\left(t, t_{0}, x_{0}\right)$ is a solution of (2) with $x_{0} \in \mathbb{R}^{n}$. The function $T$ is called the settling-time function of the system (2).

Definition 2 ([22]). The origin of system (2) is said to be globally fixed-time stable if it is globally uniformly finitetime stable and the settling-time function $T$ is globally bounded by some positive number $T_{\max }>0$, i.e. $T\left(x_{0}\right) \leq$ $T_{\text {max }}, \forall x_{0} \in \mathbb{R}^{n}$.

The characterization of fixed-time stability property by means of a Lyapunov function can be found in [9], [22].

\section{B. Implicit Lyapunov Function Method}

The Implicit Lyapunov Function method allows to study the stability of a system without presenting the Lyapunov function in an explicit form. Indeed, the Lyapunov function can remain in an implicit form, for example, as a solution of some algebraic equation (e.g. $Q(V, x)=0$ ), and in order to analyze the stability of the system it is not necessary to solve this equation; it suffices to study certain of its properties alongside with the right-hand side of the system, as stated by the next theorem.

Theorem 3. [18] If there exists a continuous function $Q$ : $\mathbb{R}_{+} \times \mathbb{R}^{n} \rightarrow \mathbb{R}$ such that:

C1 $Q(V, x)$ is continuously differentiable for $\forall x \in \mathbb{R}^{n} \backslash\{0\}$ and $\forall V \in \mathbb{R}_{+}$;

C2 for any $x \in \mathbb{R}^{n} \backslash\{0\}$ there exists $V \in \mathbb{R}_{+}$such that $Q(V, x)=0$

C3 for $\Omega=\left\{(V, x) \in \mathbb{R}^{n+1}: Q(V, x)=0\right\}$ we have $\lim _{\substack{x \rightarrow 0 \\(V, x) \in \Omega}} V=0^{+}, \lim _{\substack{V \rightarrow 0^{+} \\(V, x) \in \Omega}}\|x\|=0, \quad \lim _{\substack{\|x\| \rightarrow \infty \\(V, x) \in \Omega}} V=+\infty ;$

C4 for $\forall V \in \mathbb{R}_{+}$and $\forall x \in \mathbb{R}^{n} \backslash\{0\}$ the inequality $-\infty<\frac{\partial Q(V, x)}{\partial V}<0$ holds;

C5 $\frac{\partial Q(V, x)}{\partial x} f(t, x)<0, \quad \forall t \in \mathbb{R}$ and $\forall(V, x) \in \Omega$;

then the origin of system (2) is globally asymptotically stable.

The proof of this theorem is simply based on the classical Implicit Function Theorem [23]. By means of recent results in the ILF method [24], [25], [18] it is possible to extend this result to assert finite-time stability.
Theorem 4. [18] If there exists a continuous function $Q$ : $\mathbb{R}_{+} \times \mathbb{R}^{n} \rightarrow \mathbb{R}$ that satisfies conditions $\mathbf{C 1 - C 4}$ of Theorem 3 and

C5* $^{*} \frac{\partial Q(V, x)}{\partial x} f(t, x) \leq c V^{1-\mu} \frac{\partial Q(V, x)}{\partial V}, \forall t \in \mathbb{R}, \forall(V, x) \in \Omega$, where $c>0$ and $0<\mu \leq 1$ are some constants, then the origin of system (2) is uniformly finite-time stable with the settling-time estimate $T\left(x_{0}\right) \leq \frac{V_{0}^{\mu}}{c \mu}$, where $V_{0} \in \mathbb{R}_{+}$: $Q\left(V_{0}, x_{0}\right)=0$.

The following extension of the previous theorem helps us to analyze the fixed-time stability.

Theorem 5. [18] Let two functions $Q_{1}$ and $Q_{2}$ satisfy the conditions C1-C4 of Theorem 3 and

C6 $Q_{1}(1, x)=Q_{2}(1, x)$ for all $\forall x \in \mathbb{R}^{n} \backslash\{0\}$;

C7 $\frac{\partial Q_{1}(V, x)}{\partial x} f(t, x) \leq c_{1} V^{1-\mu} \frac{\partial Q_{1}(V, x)}{\partial V}, \forall t \in \mathbb{R}, \forall V \in$ $(0,1], \forall x \in\left\{z \in \mathbb{R}^{n} \backslash\{0\}: Q_{1}(V, z)=0\right\}$, where $c_{1}>$ 0 and $0<\mu<1$ are some constants;

C8 $\frac{\partial Q_{2}(V, x)}{\partial x} f(t, x) \leq c_{2} V^{1+\nu} \frac{\partial Q_{2}(V, x)}{\partial V}, \forall t \in \mathbb{R}, \forall V \geq$ $1, \forall x \in\left\{z \in \mathbb{R}^{n} \backslash\{0\}: Q_{2}(V, z)=0\right\}$, where $c_{2}>0$ and $\nu>0$ are some constants.

Then the origin of the system (2) is fixed-time stable and $T\left(x_{0}\right) \leq \frac{1}{c_{1} \mu}+\frac{1}{c_{2} \nu}$.

The presented theorems are used below for analysis and design of finite-time and fixed-time observers. The corresponding implicit Lyapunov function candidate is selected as follows

$$
Q(V, z):=z^{T} D_{r}\left(V^{-1}\right) P D_{r}\left(V^{-1}\right) z-1
$$

where $V \in \mathbb{R}_{+}, z \in \mathbb{R}^{n}, P \in \mathbb{R}^{n \times n}$ is a symmetric positive definite matrix, i.e. $P=P^{T}>0$ and $D_{r}(\cdot)$ is the dilation matrix of the form

$$
D_{r}(\lambda)=\left(\begin{array}{cccc}
\lambda^{r_{1}} I_{n_{1}} & 0 & \ldots & 0 \\
0 & \lambda^{r_{2}} I_{n_{2}} & \ldots & 0 \\
0 & 0 & \ldots & \lambda^{r_{m}} I_{n_{m}}
\end{array}\right)
$$

with $r=\left(r_{1}, r_{2}, \ldots, r_{m}\right)^{T} \in \mathbb{R}^{m}, r_{i}>0$ and $n_{i}$ are natural numbers such that $n_{1}+\ldots+n_{m}=n$.

\section{OBSERVER DESIGN USING IMPLICIT LYAPUNOV FUNCTION METHOD}

\section{A. Finite-Time Observer}

Decomposition algorithms for observable linear systems into a canonical form can be found in many papers, e.g [26], [27], [28], [29]. We use the transformation $\Phi$ (see Appendix) such that $\Phi A \Phi^{-1}=F \tilde{C}+\tilde{A}$, where $\tilde{C}=\left[\begin{array}{ll}I_{n_{1}} & 0\end{array}\right] \in$ $\mathbb{R}^{n_{1} \times n}, F \in \mathbb{R}^{n \times n_{1}}$,

$$
\begin{gathered}
\tilde{A}=\left(\begin{array}{ccccc}
0 & A_{12} & 0 & \ldots & 0 \\
0 & 0 & A_{23} & \ldots & 0 \\
\ldots & \ldots & \ldots & \ldots & \ldots \\
0 & 0 & 0 & \ldots & A_{m-1 m} \\
0 & 0 & 0 & \ldots & 0
\end{array}\right), \\
C \Phi^{-1}=\left(\begin{array}{cccc}
C_{0} & 0 & \ldots & 0
\end{array}\right), C_{0} \in \mathbb{R}^{k \times n_{1}}
\end{gathered}
$$


$n_{1}=\operatorname{rank}(C)$ and $n_{j}=\operatorname{rank}\left(A_{j-1 j}\right), j=2, \ldots, m$. Since $C$ has full row rank, then $n_{1}=k$ and $C_{0}$ is square and nonsingular.

It is worth stressing that the canonical forms and related transformations also exist for nonlinear systems (see, e.g [30], [13]). Therefore, the observer design algorithms given below can be easily adapted to the nonlinear case.

Let us consider the following nonlinear observer

$$
\frac{d}{d t} \hat{x}(t)=A \hat{x}(t)+G_{F T}(y(t)-C \hat{x}(t))+B u(t),
$$

where $\hat{x}(t) \in \mathbb{R}^{n}$ is the observer state vector and the function $G_{F T}: \mathbb{R}^{k} \rightarrow \mathbb{R}^{n}$ is defined as

$$
G_{F T}(\sigma)=-\Phi^{-1}\left[D_{\tilde{r}}\left(\left\|C_{0}^{-1} \sigma\right\|^{-1}\right) L-F\right] C_{0}^{-1} \sigma, \quad \sigma \in \mathbb{R}^{k}
$$

where the matrix $\Phi \in \mathbb{R}^{n \times n}$ is defined by (32), the matrix $C_{0}$ is defined by (31), the matrix $F$ is defined by (5), $D_{\tilde{r}}(\cdot)$ is the dilation matrix given by (4) with

$$
\tilde{r}=\left(\frac{\mu}{1+(m-1) \mu}, \frac{2 \mu}{1+(m-1) \mu}, \ldots, \frac{m \mu}{1+(m-1) \mu}\right)^{T}
$$

and the matrix of the observer gains $L \in \mathbb{R}^{n \times n_{1}}$ is to be defined.

Let the observation error variable be defined as $e=\Phi(x-$ $\hat{x})$. The error equation has the form

$$
\dot{e}=\left(\tilde{A}+D_{\tilde{r}}\left(\|\tilde{C} e\|^{-1}\right) L \tilde{C}\right) e,
$$

where $\tilde{A} \in \mathbb{R}^{n \times n}$ and $\tilde{C} \in \mathbb{R}^{n_{1} \times n}$ are defined by (5). Obviously, if $\mu \rightarrow 0$ then $D_{\tilde{r}}\left(\|\tilde{C} e\|^{-1}\right) \rightarrow I_{n}$ and the presented observer becomes the classical Luenberger one.

Let us denote

$$
\Xi(\lambda)=\lambda\left(D_{\tilde{r}}(1 / \lambda)-I_{n}\right), \quad \lambda \geq 0 .
$$

$r=\left[1+\frac{\mu}{1+(m-1) \mu}\right](1, \ldots, 1)^{T}-\tilde{r} \in \mathbb{R}_{+}^{m}$ and $H_{r}=$ $\operatorname{diag}\left(r_{1} I_{n_{1}}, r_{2} I_{n_{2}}, \ldots, r_{m} I_{n_{m}}\right) \in \mathbb{R}^{n \times n}$.

Theorem 6. Let for some $\mu>0$ and some $\delta \in(0,1)$ the system of matrix inequalities

$$
\begin{gathered}
\left(\begin{array}{cc}
P \tilde{A}+\tilde{A}^{T} P+\tilde{C}^{T} Y^{T}+Y \tilde{C}+P+P H_{r}+H_{r} P & P \\
P & -Z
\end{array}\right) \leq 0 \\
\left(\begin{array}{cc}
I_{k} & Y^{T} \\
Y & P
\end{array}\right) \geq 0, \quad P>0 \\
P H_{r}+H_{r} P>0, \quad P \geq \delta \tilde{C}^{T} \tilde{C} P \tilde{C}^{T} \tilde{C}, \\
Z>0, \quad \Xi(\lambda) Z \Xi(\lambda) \leq P, \quad \forall \lambda \in\left[0, \delta^{-1 / 2}\right],
\end{gathered}
$$

be feasible with $P, Z \in \mathbb{R}^{n \times n}$ and $Y \in \mathbb{R}^{n_{1} \times n}$ then the error equation (9) with $L=P^{-1} Y$ is globally finite-time stable and the settling time $T$ admits the estimate: $T \leq$ $\frac{1+(m-1) \mu}{\mu} V^{\frac{\mu}{1+(m-1) \mu}}(e(0))$, where $V: \mathbb{R}^{n} \rightarrow \mathbb{R}$ is defined implicitly by the equation $Q(V, \cdot)=0$ with $Q$ given by (3).

In other words, this theorem claims that any solution of the observer system (6) converges to a solution of the real system (1) in a finite time $T$, which is dependent on the initial estimation error $e(0) \in \mathbb{R}^{n}$. The main idea of the proof is to show that the function $Q$ (defined in the statement of
Theorem 6) satisfies all conditions of Theorem 4. The proofs in this work are skipped due to space restrictions.

Corollary 7. The system of matrix inequalities (11)-(14) is feasible for sufficiently small $\mu>0$.

Indeed, observability of the pair $(A, C)$ implies that the pair $\left(\tilde{A}+H_{r}+0.5 I_{n}, \tilde{C}\right)$ is also observable. Hence, it can be easily shown that the system of LMIs (11)-(12) is feasible with some positive definite matrix $P \in \mathbb{R}^{n \times n}, Y \in \mathbb{R}^{n_{1} \times n}$ and $Z=\alpha I_{n}$ for sufficiently large $\alpha>0$. The matrix inequalities (13) are obviously feasible for sufficiently small $\delta, \mu \in(0,1)$. Since $\|\Xi(\lambda)\| \rightarrow 0$ uniformly on $\lambda \in\left[0, \delta^{-1 / 2}\right]$ as $\mu \rightarrow 0$, then the inequality (14) will also hold for sufficiently small $\mu>0$.

In order to apply Theorem 6 in practice we need to solve the parametrized system of nonlinear matrix inequalities (11)-(14) with respect to variables $P, Z, Y$ and $\delta$ for a given $\mu \in(0,1)$. If $\delta \in(0,1)$ and $\lambda \in\left[0, \delta^{-1 / 2}\right]$ are fixed, then the system (11)-(14) becomes an LMI, which can be solved using any appropriate mathematical software (e.g. MATLAB). However, the mentioned LMI must be checked for any $\lambda \in\left[0, \delta^{-1 / 2}\right]$. Due to the smoothness of $\Xi$ with respect to $\lambda \in \mathbb{R}_{+}$, this can be done on a proper grid constructed over the interval $\left[0, \delta^{-1 / 2}\right]$. The next corollary provides sufficient feasibility condition of the parametrized matrix inequality (14).

Proposition 8. The parametric matrix inequality (14) holds if for some $\mu \in(0,1]$ and $\delta \in(0,1)$

$$
\begin{gathered}
\Xi\left(\lambda_{i}\right) Z \Xi\left(\lambda_{i}\right)+\left(\lambda_{i}-\lambda_{i-1}\right) M<P, \quad i=1, \ldots, N, \\
P>0, \quad Z>0, \\
\left(\begin{array}{cc}
\left(I_{n}-H_{\tilde{r}}\right) Z+Z\left(I_{n}-H_{\tilde{r}}\right) & -Z H_{\tilde{r}} \\
-H_{\tilde{r}} Z & M
\end{array}\right)>0, \quad M>0,
\end{gathered}
$$

where $0=\lambda_{0}<\lambda_{1}<\ldots<\lambda_{N}=\delta^{-1 / 2}, H_{\tilde{r}}=$ $\operatorname{diag}\left(\tilde{r}_{1} I_{n_{1}}, \tilde{r}_{2} I_{n_{2}}, \ldots, \tilde{r}_{m} I_{n_{m}}\right), P, M, Z \in \mathbb{R}^{n \times n}$.

The provided result allows to implement a very simple algorithm to solve the parametrized system of matrix inequalities (11)-(14) with fixed $\delta$ and $\mu$.

\section{Algorithm 9. \\ Initialization : $p=0, N=1, \lambda_{0}=0, \lambda_{N}=\delta^{-1 / 2}, \Sigma_{0}=$} $\left\{\lambda_{0}, \lambda_{N}\right\}$

Loop : While the system of LMIs (11), (12), (15) with $\lambda_{i} \in \Sigma_{p}$ is not feasible, do $\Sigma_{p+1}=\Sigma_{p} \cup\left\{\frac{\lambda_{i-1}+\lambda_{i}}{2}\right\}_{i=1}^{N}$ with $\lambda_{i} \in \Sigma_{p}, N \leftarrow 2 N$ and $p \leftarrow p+1$.

Since the matrix inequality $\left(I_{n}-H_{\tilde{r}}\right) Z+Z\left(I_{n}-H_{\tilde{r}}\right)>0$ is obviously feasible for sufficiently small $\mu>0$, then in the view of Corollary 7 the presented algorithm always finds the required solution if $\mu$ is sufficiently small.

\section{B. Fixed-Time Observer}

Let us consider the following observer

$$
\frac{d}{d t} \hat{x}(t)=A \hat{x}+G_{F x T}(y(t)-C \hat{x}(t))+B u(t),
$$


where, as before, $\hat{x} \in \mathbb{R}^{n}$ is the vector of the observer state and the function $G_{F x T}: \mathbb{R}^{k} \rightarrow \mathbb{R}^{n}$ is defined as follows

$$
\begin{gathered}
G_{F x T}(\sigma)= \\
-\Phi^{-1}\left[\frac{1}{2}\left\{D_{\tilde{r}}\left(\left\|C_{0}^{-1} \sigma\right\|^{-1}\right)+D_{\tilde{r}}\left(\left\|C_{0}^{-1} \sigma\right\|\right)\right\} L-F\right] C_{0}^{-1} \sigma,
\end{gathered}
$$

the matrix $\Phi \in \mathbb{R}^{n \times n}$ is defined by (32), the matrix $C_{0}$ is defined by (31), the matrix $F$ is defined by (5), $L \in \mathbb{R}^{n \times n_{1}}$ are the matrices of observer gains to be defined and $\sigma \in \mathbb{R}^{n_{1}}$.

The error equation for $e=\Phi(x-\hat{x})$ has the form

$$
\dot{e}=\left(\tilde{A}+\frac{1}{2}\left\{D_{\tilde{r}}\left(\|\tilde{C} e\|^{-1}\right)+D_{\tilde{r}}(\|\tilde{C} e\|)\right\} L \tilde{C}\right) e
$$

where, as before, $\tilde{A} \in \mathbb{R}^{n \times n}$ and $\tilde{C} \in \mathbb{R}^{n_{1} \times n}$ are defined by (5). Let us denote

$$
\begin{gathered}
r_{i}=(-1)^{i} \tilde{r}+\left[1+\frac{(-1)^{i+1} \mu}{1+(m-1) \mu}\right](1, \ldots, 1)^{T}, \\
H_{i}=\operatorname{diag}\left(\left(r_{i}\right)_{1} I_{n_{1}},\left(r_{i}\right)_{2} I_{n_{2}}, \ldots,\left(r_{i}\right)_{m} I_{n_{m}}\right) \\
\bar{\Xi}_{i}(\lambda, \gamma)=\frac{\lambda}{2}\left\{D_{\tilde{r}}\left(\frac{\gamma^{i-1}}{\lambda}\right)+D_{\tilde{r}}\left(\frac{\lambda}{\gamma^{i-2}}\right)-2 I_{n}\right\} . \\
\lambda>0, \quad \gamma>0, \quad i=1,2 .
\end{gathered}
$$

Theorem 10. Let for some $\mu \in(0,1)$ and $\alpha>0$ the system of matrix inequalities

$$
\begin{gathered}
\left(\begin{array}{c}
P \tilde{A}+\tilde{A} P+\tilde{C} Y^{T}+Y \tilde{C}+\alpha\left(P+P H_{i}+H_{i} P\right) \\
P
\end{array} \begin{array}{c}
P \\
-Z_{i}
\end{array}\right) \leq 0 \\
\left(\begin{array}{cc}
\alpha I_{k} & Y^{T} \\
Y & P
\end{array}\right) \geq 0, \quad P>0 \\
P H_{i}+H_{i} P>0, \quad P \geq \delta \tilde{C}^{T} \tilde{C} P \tilde{C}^{T} \tilde{C}, \quad 0<\delta<1, i=1,2 \\
Z_{i}>0, \quad \bar{\Xi}_{i}(\lambda, \gamma) Z_{i} \bar{\Xi}_{i}(\lambda, \gamma) \leq P \\
\forall \lambda \in\left(0, \delta^{-1 / 2}\right], \forall \gamma \in(0,1]
\end{gathered}
$$

be feasible with $P, Z_{1}, Z_{2} \in \mathbb{R}^{n \times n}, Y \in \mathbb{R}^{n_{1} \times n}$ then the error equation (18) with $L=P^{-1} Y$ is globally fixed-time stable with $T_{\max } \leq 2 \frac{1+(m-1) \mu}{\alpha \mu}$.

The proof of this theorem is based on the implicit Lyapunov function method and Theorem 5 .

Similarly to Corollary 7 it can be shown that the system of matrix inequalities (21)-(24) is feasible for sufficiently small $\mu \in(0,1)$.

Under additional restriction to the matrices $Z_{i}$ the parametric matrix inequality (24) can be simplified.

Proposition 11. Let $0=\lambda_{0}<\lambda_{1}<\ldots<\lambda_{N_{1}}=\delta^{-1 / 2}$ and $0=\gamma_{0}<\gamma_{1}<\ldots<\gamma_{N_{2}}=1$ for some fixed $\delta \in(0,1)$. If the matrices $S_{i}, Z_{i}, R_{i}, M_{i}, U_{i} \in \mathbb{R}^{n \times n}$ and the number $\beta>0$ satisfy the following LMIs

$$
\begin{gathered}
S_{i} H_{\tilde{r}}+H_{\tilde{r}} S_{i}>0, \quad S_{i}>0 \\
\left(\begin{array}{ccc}
2 Z_{i}-Z_{i} H_{\tilde{r}}-H_{\tilde{r}} Z_{i} & 2 Z_{i}+Z_{i} H_{\tilde{r}}-H_{\tilde{r}} Z_{i} & 2 Z_{i}-H_{\tilde{r}} Z_{i} \\
2 Z_{i}-Z_{i} H_{\tilde{r}}+H_{\tilde{r}} Z_{i} & 2 Z_{i}+Z_{i} H_{\tilde{r}}+H_{\tilde{r}} Z_{i}+S_{i} & 2 Z_{i}+H_{\tilde{r}} Z_{i} \\
2 Z_{i}-Z_{i} H_{\tilde{r}} & 2 Z_{i}+Z_{i} H_{\tilde{r}} & 2 Z_{i}+R_{i}
\end{array}\right) \geq 0 \\
R_{i}>0
\end{gathered}
$$

$$
\left(\begin{array}{cc}
Z_{i} H_{\tilde{r}}+H_{\tilde{r}} Z_{i}-\beta Z_{i} & H_{\tilde{r}} Z_{i}-\beta Z_{i} \\
Z_{i} H_{\tilde{r}}-\beta Z_{i} & M_{i}-\beta Z_{i}
\end{array}\right) \geq 0, \quad M_{i}>0
$$

$$
\begin{gathered}
\left(\begin{array}{cc}
2 M_{i}+(-1)^{i}\left(H_{\tilde{r}} M_{i}+M_{i} H_{\tilde{r}}\right) & 2 M_{i}+(-1)^{i} H_{\tilde{r}} M_{i} \\
2 M_{i}+(-1)^{i} M_{i} H_{\tilde{r}} & U_{i}
\end{array}\right) \geq 0 \\
U_{i}>0 \\
\bar{\Xi}_{i}\left(\lambda_{j}, \gamma_{s}\right) Z_{i} \Xi_{i}\left(\lambda_{j}, \gamma_{s}\right)+\left(\lambda_{j}-\lambda_{j-1}\right) R_{i}+ \\
\quad \frac{\lambda_{j}-\lambda_{j-1}}{4} D_{\tilde{r}}\left(\frac{\lambda_{j}}{\gamma_{s}^{i-2}}\right) S_{i} D_{\tilde{r}}\left(\frac{\lambda_{j}}{\gamma_{s}^{i-2}}\right)+ \\
\frac{\gamma_{s}^{\beta}-\gamma_{s-1}^{\beta}}{\beta \gamma_{s}^{\beta}}\left(\bar{\Xi}_{i}\left(\lambda_{j}, 0\right) M_{i} \bar{\Xi}_{i}(\lambda, 0)+\left(\lambda_{j}-\lambda_{j-1}\right) U_{i}\right) \leq P \\
i=1,2, \quad j=1,2, \ldots, N_{1}, \quad s=1,2, \ldots, N_{2}
\end{gathered}
$$

then (24) holds.

Based on this proposition and analogously with the finitetime case, we can provide a simple algorithm for parameter tuning for the fixed-time observer.

\section{Algorithm 12.}

Initialization : $p=0, N=1, \lambda_{0}=0, \lambda_{N}=\delta^{-1 / 2}, \quad \Sigma_{0}=$ $\left\{\lambda_{0}, \lambda_{N}\right\}, \gamma_{0}=0, \gamma_{N}=1, \Gamma_{0}=\left\{\gamma_{0}, \gamma_{N}\right\}$.

Loop : While the system of LMIs (11), (12), (15) with $\lambda_{i} \in \Sigma_{p}$ is not feasible, do $\Sigma_{p+1}=\Sigma_{p} \cup\left\{\frac{\lambda_{j-1}+\lambda_{j}}{2}\right\}_{j=1}^{N}$ with $\lambda_{j} \in \Sigma_{p}$,

$\Gamma_{p+1}=\Gamma_{p} \cup\left\{\frac{\gamma_{s-1}+\gamma_{s}}{2}\right\}_{s=1}^{N}$ with $\gamma_{s} \in \Gamma_{p}$,

$N \leftarrow 2 N$ and $p \leftarrow p+1$.

\section{EXAMPLES}

\section{A. Finite-time observer}

Let us consider the linear system (1) with parameters

$$
\begin{gathered}
A=\left(\begin{array}{ccccc}
1 & 1 & 2 & 4 & 5 \\
1 & 3 & 2 & -1 & -3 \\
2 & 1 & -3 & 7 & 3 \\
4 & -2 & 9 & 1 & -1 \\
1 & -1 & 0 & 2 & 1
\end{array}\right), B=\left(\begin{array}{l}
0 \\
0 \\
0 \\
0 \\
1
\end{array}\right) \\
C=\left(\begin{array}{ccccc}
1 & 0 & 0 & 0 & 0 \\
0 & 0 & 0 & 0 & 1
\end{array}\right)
\end{gathered}
$$

First, we transform the system to the observable canonical form by using the matrix

$$
\Phi=\left(\begin{array}{ccccc}
1.0000 & 0 & 0 & 0 & 0 \\
0 & 0 & 0 & 0 & 1.0000 \\
-1.2333 & -0.0793 & 0.2673 & 0.9604 & 2.6729 \\
0.8995 & -0.8414 & -0.5345 & 0.0793 & 0.3865 \\
-0.2083 & -0.9068 & 0.8159 & 0.5198 & -3.1524
\end{array}\right)
$$

Then, by applying Algorithm 9 for $\mu=1$ and $\delta=\frac{1+\sqrt{5}}{3+\sqrt{5}}$ we find the observer gains matrix as

$$
L=\left(\begin{array}{ll}
-5.2009 & -0.9657 \\
-0.8583 & -4.6400 \\
-7.6469 & -6.6227 \\
-0.4210 & -7.7855 \\
-2.0775 & -1.4736
\end{array}\right)
$$

Fig. 1 presents the simulation results for finite-time observer (6) for $u(t)=\sin (t)$ and $\hat{x}(0)=0 \in \mathbb{R}^{5}, x(0)=$ $\left(\begin{array}{lllll}0.1 & -0.1 & 0.1 & -0.1 & 0.1\end{array}\right)^{T}$. Note that the considered linear system is unstable (in particular, $\|x(1)\| \approx 10^{7}$ ), but the convergence of the observed state to the real state of the linear system is guaranteed in a finite-time. 


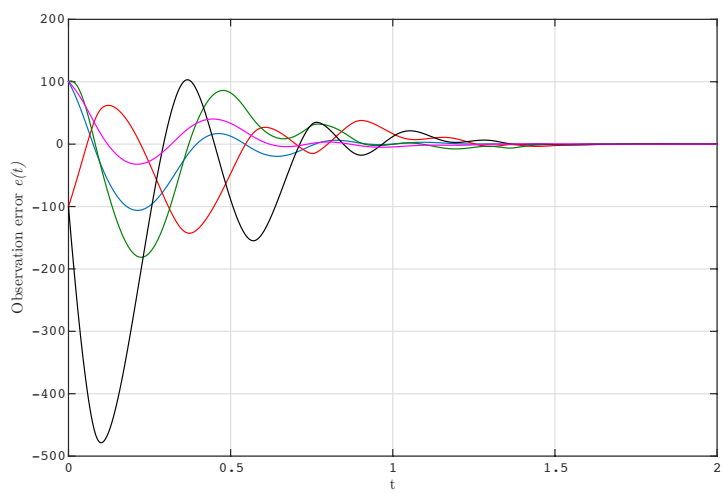

Fig. 1. Evolution of the observation error for finite-time observer

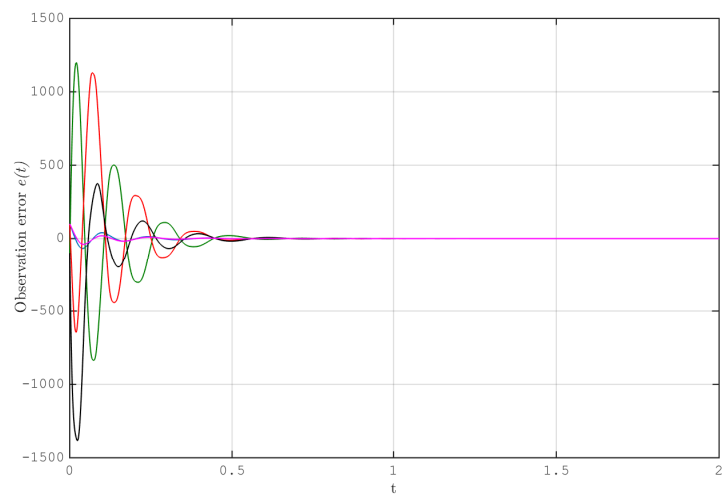

Fig. 2. Evolution of the observation error for fixed-time observer

\section{B. Fixed-time observer}

The fixed-time observer is designed by first setting $\mu=$ 0.5 , then, according to (10), to predefined a convergence time of $T_{\max } \leq 1.75, \alpha=32 / 7$. The resulting observer gain matrix is

$$
L=\left(\begin{array}{cc}
-22.9151 & -4.7168 \\
-4.8386 & -19.1328 \\
-170.0681 & -138.8725 \\
-17.5165 & -152.9384 \\
-234.9672 & -159.6799
\end{array}\right)
$$

Fig. 2 depicts the simulation results of fixed-time observer application to linear system (1) with input signal $u(t)=$ $\sin (t)$ and the initial condition $x(0)=10^{2} x^{*}, x^{*}=$ $\left(\begin{array}{lllll}1 & -1 & 1 & -1 & 1\end{array}\right)^{T}$. The simulations have been also done for initial conditions : $x(0)=10^{3} x^{*}$ and $x(0)=10^{4} x^{*}$. In all three cases (Independently of the initial condition) the obtained estimation error is $\|e(1.75)\| \leq 2 \cdot 10^{-4}$. Note that the finite-time observer given above is not appropriate for large initial errors, since it provides $\|e(1.75)\| \geq 100$ for $x(0)=10^{3} x^{*}$.

\section{CONCLUSION}

This paper presents finite-time and fixed-time observers for linear systems as well as LMI-based algorithms for tuning the observers gains. Since the design is based on a particular transformation to canonical observability form, similar observers can be easily applied to non-linear systems that admit this canonical form. The optimal tuning of the observers parameters in the case of noise in the measurements and system uncertainties is left for future research.

\section{APPENDIX}

\section{A. Block Decomposition}

Let the matrices $T_{i}$ be defined by the following algorithm:

Initialization : $A_{1}=A, C_{1}=C, T_{1}=I_{n}, m=1$.

Loop: While $\operatorname{rank}\left(C_{m}\right)<\operatorname{rown}\left(A_{m}\right)$ do

$$
\begin{aligned}
& A_{m+1}=\left(C_{m}^{\perp}\right)^{T} A_{m} C_{m}^{\perp}, \\
& C_{m+1}=\hat{C}_{m}^{T} A_{m} C_{m}^{\perp}, \\
& T_{m+1}=\left(\begin{array}{cc}
\hat{C}_{m} & C_{m}^{\perp}
\end{array}\right), \quad m=m+1,
\end{aligned}
$$$$
\text { where } C_{m}^{\perp}:=\operatorname{null}\left(C_{m}\right), \hat{C}_{m}:=\operatorname{null}\left(\left(C_{m}^{\perp}\right)^{T}\right) \text {. }
$$

This simple algorithm can be easily realized in MATLAB. It helps to construct an orthogonal coordinate transformation of the original system (1) to the block form. If the pair $(A, C)$ is observable then the algorithm given above stops after $m$ steps, where $m<n$, and the matrix

$$
\begin{gathered}
\mathcal{O}=T_{1}\left(\begin{array}{cc}
I_{w_{2}} & 0 \\
0 & T_{2}
\end{array}\right)\left(\begin{array}{cc}
I_{w_{3}} & 0 \\
0 & T_{3}
\end{array}\right) \ldots\left(\begin{array}{cc}
I_{w_{m}} & 0 \\
0 & T_{m}
\end{array}\right), \\
\text { where } w_{i}:=n-\operatorname{rown}\left(T_{i}\right),
\end{gathered}
$$

is an orthogonal matrix such that $\mathcal{O}^{T} \mathcal{O}=\mathcal{O O}^{T}=I_{n}$ and

$$
\begin{gathered}
\mathcal{O} A \mathcal{O}^{T}=\left(\begin{array}{ccccc}
A_{11} & A_{12} & 0 & \ldots & 0 \\
A_{21} & A_{22} & A_{23} & \ldots & 0 \\
\ldots & \ldots & \ldots & \ldots & \ldots \\
A_{m-11} & A_{m-12} & \ldots & A_{m-1} m-1 & A_{m-1 m} \\
A_{m 1} & A_{m 2} & \ldots & A_{m m-1} & A_{m m}
\end{array}\right), \\
C \mathcal{O}=\left(\begin{array}{cccc}
C_{0} & 0 & \ldots & 0
\end{array}\right),
\end{gathered}
$$

where $C_{0}=C \hat{C}_{1}, A_{i j} \in \mathbb{R}^{n_{i} \times n_{j}}, n_{i}:=\operatorname{rank}\left(C_{i}\right)$, $i, j=1,2, \ldots, m$ and $\operatorname{rank}\left(A_{i+1}\right)=n_{i+1}$. This can be proven, for example, using the ideas of duality and the Lemma 3 from [22]. Since $\operatorname{rank}\left(A_{i i+1}\right)=n_{i+1}=$ $\operatorname{rown}\left(A_{i i+1}^{T}\right)$ then $A_{i i+1}^{T} A_{i i+1}$ is invertible and $A_{i i+1}^{+}=$ $\left(A_{i i+1}^{T} A_{i i+1}\right)^{-1} A_{i i+1}^{T}$ is the left inverse matrix to $A_{i i+1}$. Let us define the following matrices $A_{i j}^{[m]}=A_{i j}, i, j=$ $1,2, . ., m ; A_{q-1 q-p-1}^{[q-1]}=A_{q-1 q-p-1}^{[q]}+A_{q-1 q}^{[q]} \cdot A_{q q-p}^{[q-p]}$. $A_{q-p-1 q-p}^{+}$and $A_{q j}^{[q-p-1]}=A_{q j}^{[q-p]}-A_{q q-p}^{[q-p]} \cdot A_{q-p-1 q-p}^{+}$. $A_{q-p-1 j}^{[q-p]}$ for $q=m, m-1, \ldots, 2, p=0,1, \ldots, q-2$ and $j=1,2, \ldots, q-p-1$. It can be shown that the transformation

$\Phi=\left(\begin{array}{ccccc}I_{n_{1}} & 0 & \ldots & 0 & 0 \\ -A_{22}^{[2]} A_{12}^{+} & I_{n_{2}} & \ldots & 0 & 0 \\ \ldots & \ldots & \ldots & \ldots & \ldots \\ -A_{m-1}^{[2]}{ }_{2} A_{12}^{+} & -A_{m-13}^{[3]} A_{23}^{+} & \ldots & I_{n_{m-1}} & 0 \\ -A_{m 2}^{[2]} A_{12}^{+} & -A_{m 3}^{[3]} A_{23}^{+} & \ldots & -A_{m m}^{[m]} A_{m-1}^{+} & I_{n_{m}}^{+}\end{array}\right)^{+32)}$,

reduces the matrix $A$ to the block form: $\Phi A \Phi^{-1}=F \tilde{C}+$ $\tilde{A}$, where 


$$
\begin{gathered}
\mathrm{F}=\left(A_{11}^{[1]}, A_{21}^{[1]}, \ldots, A_{m-11}^{[1]}, A_{m 1}^{[1]},\right)^{T}, \quad \tilde{C}=\left[I_{n_{1}} 0\right] \in \mathbb{R}^{n_{1} \times n}, \\
\tilde{A}=\left(\begin{array}{ccccc}
0 & A_{12} & 0 & \ldots & 0 \\
0 & 0 & A_{23} & \ldots & 0 \\
\ldots & \ldots & \ldots & \ldots & \ldots \\
0 & 0 & 0 & \ldots & A_{m-1 m} \\
0 & 0 & 0 & \ldots & 0
\end{array}\right) .
\end{gathered}
$$

\section{REFERENCES}

[1] R. Kalman, "A new approach to linear filtering and prediction problems," Journal of Basic Engineering -, vol. 82, no. 1, pp. 35-45, 1960.

[2] D. Luenberger, "Observing the state of a linear system," IEEE Transactions on Military Electronics, vol. 8, no. 2, pp. 74-80, 1964.

[3] V. Andrieu, L. Praly, and A. Astolfi, "High-gain observers with updated high-gain and homogeneous correction terms," Automatica, vol. 45, no. 2, pp. 422-428, 2009.

[4] M. Ghanes, J. De Leon, and J.-P. Barbot, "Observer design for nonlinear systems under unknown time-varying delays," IEEE Transactions on Automatic Control, vol. 58, no. 6, pp. 1529-1534, 2013.

[5] F. Mazenc and O. Bernard, "Interval observers for linear time-invariant systems with disturbances," Automatica, vol. 47, no. 1, pp. 140-147, 2011.

[6] A. Zolghadri, D. Henry, J. Cieslak, D. Efimov, and P. Goupil, Fault Diagnosis and Fault-Tolerant Control and Guidance for Aerospace Vehicles. Springer, 2014.

[7] W. Lahoz, B. Khattatov, and R. Menard, Eds., Data Assimiliation: Making Sense of Observations. Springer, 2010.

[8] R. Engel and G. Kreisselmeier, "A continuous-time observer which converges in finite time," IEEE Transactions on Automatic Control, vol. 47, pp. 1202-1204, 2002.

[9] V. Andrieu, L. Praly, and A. Astolfi, "Homogeneous approximation, recursive obsertver and output feedback," SIAM Journal of Control and Optimization, vol. 47(4), pp. 1814-1850, 2008.

[10] W. Perruquetti, T. Floquet, and E. Moulay, "Finite-time observers: Application to secure communication," IEEE Transactions on Automatic Control, vol. 53(1), pp. 356-360, 2008.

[11] Y. Aoustin, C. Chevallereau, and Y. Orlov, "Finite time stabilization of a perturbed double integrator - part ii: applications to bipedal locomotion," in Conference on Decision and Control, 2010, pp. 35543559.

[12] T. Floquet, C. Edwards, and S. Spurgeon, "On sliding mode observers for systems with unknown inputs," International Journal of Adaptive Control and Signal Processing, vol. 21, no. 8-9, pp. 638-656, 2007.

[13] H. K. Khalil and L. Praly, "High-gain observers in nonlinear feedback control," International Journal of Robust and Nonlinear Control, vol. 24, no. 6, pp. 993-1015, 2014.

[14] A. Levant, "Robust exact differentiation via sliding mode technique," Automatica, vol. 34, no. 3, pp. 379-384, 1998.

[15] E. Cruz-Zavala, J. Moreno, and L. Fridman, "Uniform robust exact differentiator," IEEE Transactions on Automatic Control, vol. 56, no. 11 , pp. $2727-2733,2011$.

[16] M. Angulo, J. Moreno, and L. Fridman, "Robust exact uniformly convergent arbitrary order differentiator," Automatica, vol. 49, no. 8, pp. 2489-2495, 2013.

[17] S. Boyd, E. Ghaoui, E. Feron, and V. Balakrishnan, Linear Matrix Inequalities in System and Control Theory. Philadelphia: SIAM, 1994.

[18] A. Polyakov, D. Efimov, and W. Perruquetti, "Finite-time and fixedtime stabilization: Implicit Lyapunov function approach," Automatica, vol. 51, pp. 332-340, 2015.

[19] E. Roxin, "On finite stability in control systems," Rendiconti del Circolo Matematico di Palermo, vol. 15(3), pp. 273-283, 1966.

[20] S. Bhat and D. Bernstein, "Finite-time stability of continuous autonomous systems," SIAM Journal of Control and Optimization, vol. 38(3), pp. 751-766, 2000.

[21] Y. Orlov, "Finite time stability and robust control synthesis of uncertain switched systems," SIAM Journal of Control and Optimization, vol. 43(4), pp. 1253-1271, 2005.

[22] A. Polyakov, "Nonlinear feedback design for fixed-time stabilization of linear control systems," IEEE Transactions on Automatic Control, vol. 57, no. 8, pp. 2106-2110, 2012.

[23] R. Courant and F. John, Introduction to calculus and analysis (Vol. II/1). New York: Springer, 2000.
[24] V. Korobov, "A general approach to synthesis problem," Doklady Academii Nauk SSSR, vol. 248, pp. 1051-1063, 1979.

[25] J. Adamy and A. Flemming, "Soft variable-structure controls: a survey," Automatica, vol. 40, pp. 1821-1844, 2004.

[26] W. M. Wonham, Linear Multivariable Control: A Geometric Approach. Springer, 1985.

[27] S. V. Drakunov, D. B. Izosimov, A. G. Luk'yanov, V. A. Utkin, and V. I. Utkin, "The block control design principle i," Automation and Remote Control, vol. 51, no. 5, pp. 601-608, 1990.

[28] _ - "The block control design principle ii," Automation and Remote Control, vol. 51, no. 6, pp. 737-746, 1990.

[29] M. Misrikhanov and V. Ryabchenko, "Pole placement for controlling a large scale power system," Automation and Remote Control, vol. 72, no. 10 , pp. 2123-2146, 2011.

[30] A. Isidori, Nonlinear Control Systems. Springer-Verlag, N. Y. Inc., 1995. 